\title{
Entrenamiento Aeróbico y el Consumo Máximo de Oxígeno (Vo2máx) en árbitros profesionales de fútbol
}

\author{
Aerobic training and maximum oxygen consumption (VO2 max) in \\ professional football referees
}

Luis Alfredo Jiménez Ruiz. ${ }^{1}$, Juan Gabriel Caguana Caguana. ${ }^{2}$, Santiago Ernesto Garcés

Duran. ${ }^{3} \&$ Ana Gabriela Calderón Sánchez. ${ }^{4}$

Recibido: 10-02-2019 / Revisado: 15-02-209 /Aceptado: 04-03-2019/ Publicado: 14-06-2019

\begin{abstract}
.
DOI: https://doi.org/10.33262/cienciadigital.v3i2.5.539

It is important that the referee applying the rules of football today has an optimal performance throughout the performance of the game. Motivation, mental and physical lucidity allow the judge to make the best refereeing decision in a clear and credible way. The objective of this study is to know the aerobic training and the maximum consumption of oxygen (vo2max) of the professional soccer referees. 49 referees from the Tungurahua association of professional referees participated in the study, which were submitted to an evaluation through the resistance test of Course Navette in two stages, a pre and a post, with the application of an aerobic training program, which lasted six months; for the statistical analysis a comparison of means was made using the statistical inference method of student $t$; it is concluded that there

\footnotetext{
${ }^{1}$ Universidad Técnica de Ambato, Facultad de Ciencias Humanas y de la Educación, Carrera de Cultura Física. Pedagogía de la Actividad Física y Deporte. Ambato, Ecuador. la.jimenez@uta.edu.ec

${ }^{2}$ Universidad Técnica de Ambato, Facultad de Ciencias Humanas y de la Educación, Carrera de Cultura FísicaAmbato, Ecuador. jcuaguana1079@uta.edu.ec

${ }^{3}$ Universidad Técnica de Ambato, Facultad de Ciencias Humanas y de la Educación, Carrera de Cultura FísicaPedagogía de la Actividad Física y Deporte. Ambato, Ecuador. se.garces@uta.edu.ec

${ }^{4}$ Hospital General Docente Ambato. Ambato, Ecuador. anagabrielacs07@gmail.com
} 
is an improvement in the physical condition of the referees since it is demonstrated that there is an increase in the maximum consumption of oxygen after the training period.

Keywords: Physical condition, aerobic training, VO2max, performance.

\section{Resumen.}

Es importante que el árbitro aplicando el reglamento del fútbol en la actualidad tenga un rendimiento óptimo durante todo el desempeño del partido de juego. La motivación, la lucidez mental y física permite al juez que tome la mejor decisión arbitral de forma clara y creíble. el objetivo de este estudio se centra en conocer el entrenamiento aeróbico y el consumo máximo de oxigeno (vo2máx) de los árbitros profesionales de fútbol, en el estudio participaran 49 árbitros de la asociación de árbitros profesionales de Tungurahua, los cuales fueron sometidos a una valoración mediante la prueba de resistencia de Course Navette en dos etapas, un pre y un post, con la aplicación de un programa de entrenamiento aeróbico, el cual tuvo una duración de seis meses; para el análisis estadístico se realizó una comparación de medias utilizando el método de inferencia estadística de $\mathrm{t}$ de student; se concluye que existe una mejoría en la condición física de los árbitros ya que se demuestra que existe un aumento del consumo máximo de oxigeno luego del período de entrenamiento.

Palabras claves: Condición Física, entrenamiento aérobico, vo2máx, rendimiento.

\section{Introducción.}

La preparación aeróbica del árbitro de fútbol en relación al consumo máximo de oxígeno Vo2máx es un tema de mucha discusión, ya que se relaciona con el desempeño en los encuentros deportivos, por esta razón, es muy importante conocer a través de este estudio cuales son los beneficios que tiene la preparación aeróbica en los árbitros profesionales, cuáles son sus ventajas y cómo influye en la preparación física y mental del árbitro de fútbol, de esta manera podemos conocer cuál es el máximo de oxigeno que el árbitro de fútbol presenta al momento de ser evaluado físicamente, y con esto poder tener una relación si al momento de dirigir los partidos de fútbol tiene un esfuerzo físico inmenso que le produzca el agotamiento físico, por esta razón nos hemos enfocado en verificar esta problemática y de esta manera aportar al mejoramiento del árbitro de fútbol. 
En la década de 1960, el Dr. Kenneth H. Cooper desarrolló un sistema de ejercicios para prevenir la enfermedad de la arteria coronaria. El sistema fue desarrollado en el Hospital de la Fuerza Aérea y originalmente destinado a los militares. Lo llamó "aeróbicos" en un libro del mismo nombre que fue publicado en 1968. Después de su publicación, la bailarina Jackie Sorenson desarrolló rutinas de baile que apuntaban a mejorar el ejercicio cardiovascular, que se denominó aeróbico (Casselbury, 2017); el trabajo aeróbico es aquel esfuerzo físico constante de intensidad moderada que utiliza oxígeno a un ritmo en el que el sistema cardiorrespiratorio puede reponer oxígeno en los músculos que se encuentran ejercitando; como ejemplos podemos mencionar actividades como trotar en bicicleta estacionaria o trote cadenciado para la pérdida de grasa cuando se realiza en las cantidades correctas, pero es altamente catabólica si se hace en exceso (Rivera, 2016).

De 1978 a 1987, el número de personas que realizan ejercicios aeróbicos en los Estados Unidos aumentó de un estimado de 6 millones a 19 millones, según Aerobic (2017), esto destaca la aparición una nueva variedad de ejercicios cuando Howard y Karen Schwartz desarrollaron la idea como un deporte competitivo. La Palabra Aeróbico significa literalmente al ejercicio que "involucra oxígeno, relacionado con el oxígeno o requirente de oxígeno libre", entiéndase que el ejercicio físico involucra la utilización de musculatura que requiere de energía para dicho funcionamiento y esa energía proviene del metabolismo aeróbico (Cooper, 2013)

El metabolismo aeróbico es la forma en que su cuerpo crea energía a través de la combustión de carbohidratos, aminoácidos y grasas en presencia de oxígeno. La combustión significa quemar, por lo que esto se llama quemar azúcares, grasas y proteínas para obtener energía. El metabolismo aeróbico se utiliza para la producción sostenida de energía para el ejercicio y otras funciones corporales (Norman, 2017)

El entrenamiento de ejercicio de resistencia produce numerosos efectos metabólicos y cardiovasculares. Las adaptaciones metabólicas incluyen un aumento en la capacidad oxidativa del músculo esquelético (mayor cantidad y tamaño de mitocondrias); un aumento 
en la concentración de mioglobina del músculo esquelético; una mayor capacidad de oxidar ácidos grasos para obtener energía; y un aumento en el glucógeno almacenado.

Según Braun (2013):

"El ejercicio de resistencia es cualquier actividad que usa grupos musculares grandes, puede realizarse continuamente y es de naturaleza rítmica y aeróbica. Para desarrollar y mantener la capacidad cardiovascular y la Frecuencia cardiaca (FC), este ejercicio se debe realizar a una frecuencia de 3 a 5 dias por semana, una intensidad de 60\% a 90\% de FCmáx o de 50\% a 85\% de FCmáx reserva, y una duración de 20 a 60 minutos”.

El entrenamiento físico aeróbico contribuye a la aptitud cardiovascular, porque altera beneficiosamente el perfil de riesgo de la enfermedad de la arteria coronaria. Existe una relación inversa entre la condición física y la frecuencia cardíaca en reposo, el peso corporal, el porcentaje de grasa corporal, el colesterol sérico, los triglicéridos, la glucosa y la presión arterial sistólica. Además, el entrenamiento físico aumenta la fracción de lipoproteína de alta densidad del colesterol total (Bumgardner, 2017).

Según Wilmore \& Costill (2015), el VO2máx se ha definido como: "La tasa más alta de consumo de oxígeno alcanzable durante el ejercicio máximo o exhaustivo”. A medida que aumenta la intensidad del ejercicio, también aumenta el consumo de oxígeno. Sin embargo, se llega a un punto donde la intensidad del ejercicio puede seguir aumentando sin el aumento asociado en el consumo de oxígeno.

El punto en el que las mesetas de consumo de oxígeno definen el VO2max o la capacidad aeróbica máxima de un individuo. En general, se considera el mejor indicador de la resistencia cardiorrespiratoria y la capacidad aeróbica. Sin embargo, también lo discutiremos en un momento, es más útil como un indicador del potencial aeróbico o el límite superior de una persona que como un predictor de éxito en eventos de resistencia (Rivera, 2011).

La potencia aeróbica, la capacidad aeróbica y la absorción máxima de oxígeno son todos términos que se usan indistintamente con VO2 Max. Generalmente se expresa en relación con el peso corporal porque las necesidades de oxígeno y energía difieren en relación con el 
tamaño. También se puede expresar en relación con el área de la superficie del cuerpo y esto puede ser más preciso cuando se comparan los niños y el consumo de oxígeno entre los sexos (Sport-fitness, 2014).

Acosta (2012), menciona que la parte fisiológica más importante en la generación de la actividad física y el ejercicio es la contractura muscular:

"La contracción muscular durante el ejercicio físico es posible gracias a un proceso de transformación de energía. La energía química que se almacena en los enlaces de las moléculas de los diferentes sustratos metabólicos (el ATP es la molécula intermediaria en este proceso) es transformada en energía mecánica."

Según Losnegard et al. (2012):

"El VO2máx de un individuo se prueba usando una prueba de ejercicio incremental en la que la velocidad, el gradiente o la potencia (ciclismo) aumentan de forma incremental hasta que llega al máximo el consumo de oxígeno."

Durante la prueba, la absorción de oxígeno debería aumentar linealmente a medida que aumenta la intensidad del ejercicio, luego alrededor de cierto punto el consumo de oxígeno debería comenzar a nivelarse, en este punto el consumo de oxígeno es máximo y no aumentará aún si la intensidad del ejercicio aumenta aún más, de ahí el nombre de consumo máximo de oxígeno o VO2max. En algunos casos, no habrá una meseta de consumo de oxígeno; en estos casos, el VO2max ocurrirá durante la etapa final que puede completarse con éxito durante la prueba (Acosta, 2012).

Por tanto, el objetivo de este estudio fue determinar los niveles de entrenamiento aeróbico en el consumo de Vo2máx de los árbitros profesionales de fútbol de Tungurahua.

\section{Metodologia.}

El enfoque de esta investigación, se basó en el carácter mixto, cuali-cuantitativo porque se analizó los parámetros de los test aeróbicos en el consumo V02máx., cuantitativo porque se obtuvo datos numéricos los mismos que fueron tabulados de forma estadística. 
Se utilizó la bases de datos indexadas, las cuales permitió ampliar y profundizar la conceptualización según los criterios de distintos autores, apoyados específicamente en las diferentes fuentes de investigación como: libros, revistas y el trabajo de campo porque se realizó en el lugar de los hechos, teniendo contacto directo con la realidad del problema para obtener información en concordancia con las variables del estudio.

Sujetos: Se tomó en cuenta para la conformación de la muestra de estudio a 49 árbitros de la asociación de árbitros profesionales de Tungurahua, los mismos que otorgaron su consentimiento informado, y se les dio a conocer los objetivos del estudio.

Protocolos: Se realizó inicialmente la recolección de los datos personales y las medidas antropométricas, socializando la finalidad del trabajo, posteriormente los árbitros se sometieron a la prueba de resistencia de course navette, la cual consiste en medir la resistencia aeróbica de una persona mediante un recorrido de 20 metros y debe recorrer esta distancia durante los tiempos establecidos en el test, hasta que su resistencia pueda permitirlo, el test finaliza cuando el individuo no llegue por dos ocasiones al punto de referencia, posterior a la finalización de la prueba se mide la frecuencia cardiaca para el cálculo del consumo de Vo2máx.

La fórmula para el cálculo de VO2máx es:

VO2 máximo $=132,6-(0,17 x P C)-(0,39 x E d a d)+(6,31 x S)-(3,27 x T)-(0,156 x$ FC)

PC: Peso corporal.

S: Sexo (0: mujeres, 1: hombres).

\section{T: Tiempo en minutos.}

FC: Frecuencia cardiaca.

Se realizó una primera mediación para obtener datos iniciales sobre el VO2máx, luego se sometió a los árbitros un programa de entrenamiento aeróbico estricto durante un periodo de seis meses, con una duración de tres sesiones semanales. Transcurrido el tiempo se les volvió a valorar el Consumo Máximo de Oxigeno. En función de los datos iniciales y finales se procedió al análisis estadístico realizando una comparación de medias, utilizando el método de inferencia estadística de $t$ de student. 


\section{Resultados.}

De los 41 árbitros de género masculino analizados la mayoría de los sujetos presentaron buena condición $(n=12 ; 29 \%)$; una muy buena condición física $(n=7 ; 17 \%)$ y una excelente condición física $(n=5 ; 12 \%)$; mientras que en relación al extremo de la condición física podemos ver que existe mayor índice en los valores de deficiente ( $n=17 ; 42 \%)$, por lo tanto se asume que en los árbitros hombres el 71\% presentaron valores muy bajos. Figura 1.

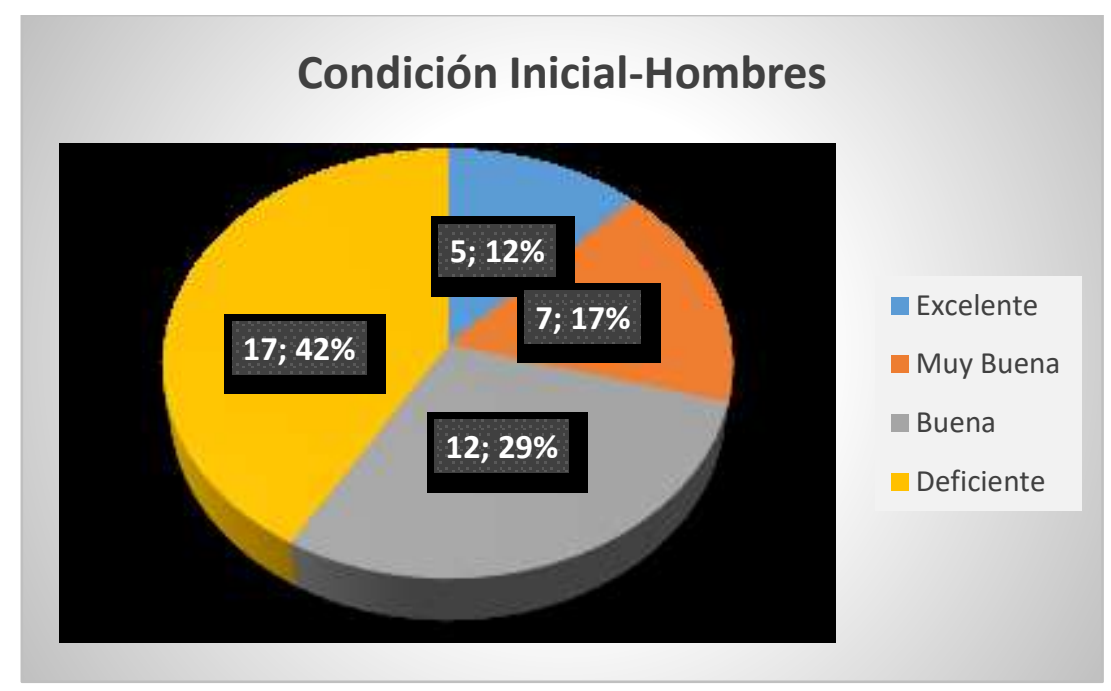

Figura 1. Condición física inicial de los Árbitros Hombres.

Fuente: Elaboración propia.

En la figura 2 se puede observar que de las 8 Mujeres Árbitros analizadas presentó (n=1; $12 \%)$ presentó una condición física excepcional, apenas $(n=1 ; 13 \%)$ presentó una condición muy buena, y 2 mujeres presentaron una condición buena $(n=2 ; 25 \%)$, mientras que en el extremo 4 mujeres árbitros obtuvieron una condición física deficiente $(n=4 ; 50 \%)$. En definitiva el $75 \%$ del grupo de mujeres presentaron valores bajos en los datos iniciales. Figura.2. 


\section{Condición Inicial-Mujeres}

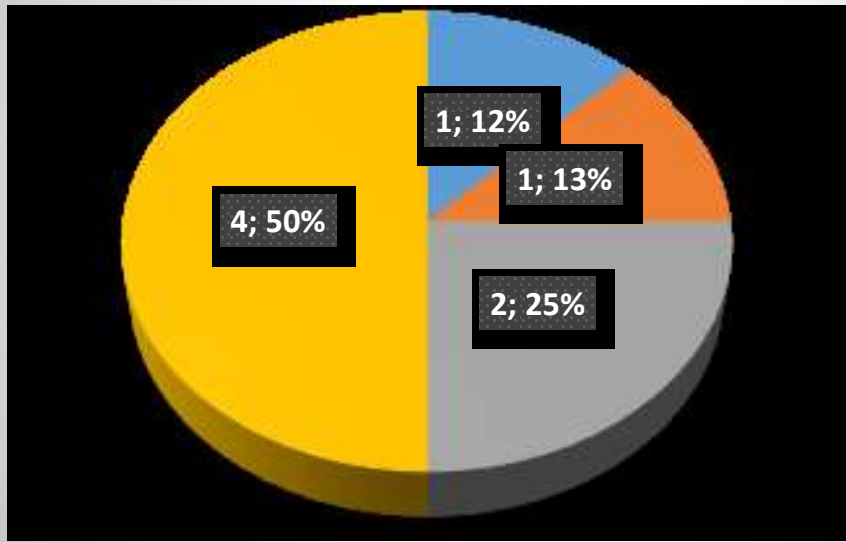

Excelente

Muy Buena

Buena

Deficiente

Figura 2. Condición física inicial de los Árbitros Mujeres.

Fuente: Elaboración propia.

Luego del programa de entrenamiento se puede visualizar a simple inspección una mejoría en la condición física de los árbitros en el género masculino ya que se demuestra que existe un aumento del consumo máximo de oxigeno luego del periodo de entrenamiento, se puede observar que existen tan solo 2 árbitros $(n=2 ; 5 \%)$, con valores deficientes; 5 árbitros $(n=5$; $12 \%)$ con buena condición; mientras que 16 árbitros hombres $(n=16 ; 39 \%)$ obtuvieron valores de muy buena, en tanto que 18 hombres $(n=18 ; 44 \%)$ llegaron a un nivel excelente dentro de su condición física. Figura 3.

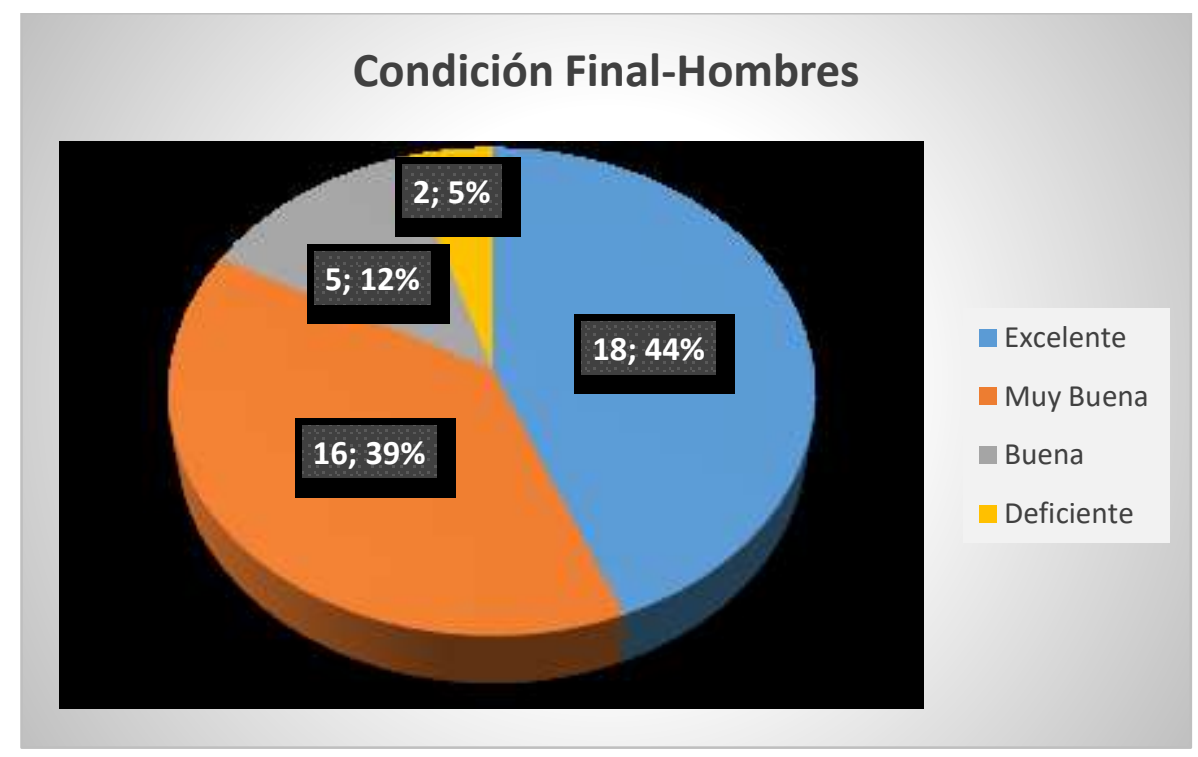


Figura 3. Condición Física final de los Árbitros Hombres.

Fuente: Elaboración propia.

Dentro del grupo de las mujeres luego del programa de entrenamiento se obtuvieron mejores valores ya que 6 árbitros mujeres ( $\mathrm{n}=6 ; 75 \%$ ) obtuvieron valores de excelente, 1 arbitro mujer $(\mathrm{n}=1 ; 12 \%)$ obtuvo muy buena condición y 1 mujer $(\mathrm{n}=1 ; 13 \%)$ registro buena condición física, y ya no se evidenció valores deficientes. Por lo tanto se pudo obtener una mejoría en el VO2máx luego del entrenamiento en el grupo de los árbitros mujeres, ya que se suprimió el grupo de árbitros con condición deficiente y aumentó el grupo de árbitros con excelente nivel en la condición física. Figura 4.

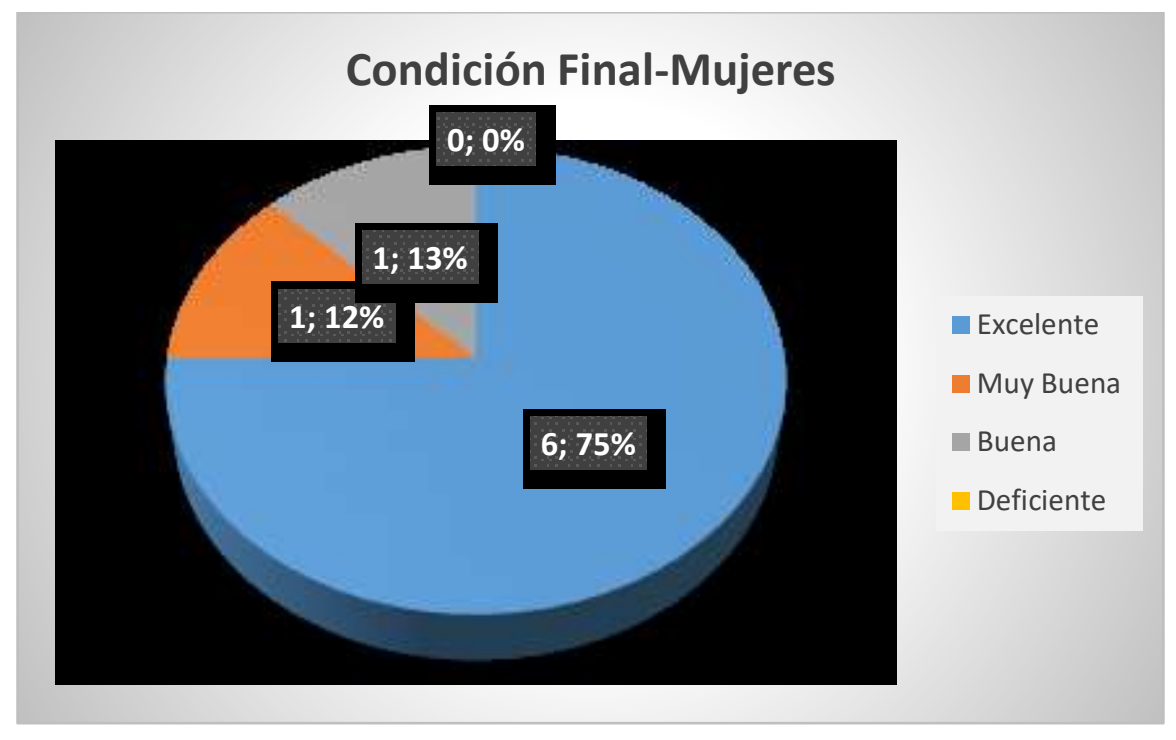

Figura 4. Condición Física final de los Árbitros Mujeres.

Fuente: Elaboración propia.

Decisión: Se demostró que existe una correlación inferencial con significancia estadística, ya que el valor $\mathrm{t}=123,7$ es $(<0,0001)$ por lo que se acepta la hipótesis: El entrenamiento aeróbico para el grupo de estudio influye directamente en la condición física.

Adicionalmente los datos encontrados se puede mencionar que mejoró el Consumo Máximo de Oxigeno como se puede observar en la tabla 1 y 2. 
Tabla 1. Promedios de los valores de VO2max antes y después del entrenamiento

\begin{tabular}{lllll} 
& $\mathrm{N}$ & Media & $\begin{array}{l}\text { Desviación } \\
\text { estándar }\end{array}$ & $\begin{array}{l}\text { Media de error } \\
\text { estándar }\end{array}$ \\
\hline vo2max 1 & 49 & 69,119 & 5,0485 &, 7212 \\
vo2max 2 & 49 & 72,841 & 4,1220 &, 5889
\end{tabular}

Fuente: Elaboración propia.

Tabla 2. Inferencia mediante t de student

Valor de prueba $=0$

\begin{tabular}{|c|c|c|c|c|c|c|}
\hline & \multirow[b]{2}{*}{$\mathrm{t}$} & \multirow[b]{2}{*}{$\mathrm{gl}$} & \multirow{2}{*}{$\begin{array}{l}\text { Sig. } \\
\text { (bilateral) }\end{array}$} & \multirow{2}{*}{$\begin{array}{l}\text { Diferencia } \\
\text { de medias }\end{array}$} & \multicolumn{2}{|c|}{$\begin{array}{l}95 \% \text { de intervalo de } \\
\text { confianza de la diferencia }\end{array}$} \\
\hline & & & & & Inferior & Superior \\
\hline Vo2max 1 & 95,836 & 48 &, 000 & 69,1190 & 67,669 & 70,569 \\
\hline Vo2max 2 & 123,700 & 48 & ,000 & 72,8409 & 71,657 & 74,025 \\
\hline
\end{tabular}

Fuente: Elaboración propia.

\section{Discusión.}

Las mejoras constantes observadas en la tolerancia al ejercicio podrían explicarse por el aumento en el VO2máx, sin embargo, existen estudios que informan una correlación consistente entre las mejoras de rendimiento aeróbico y el VO2máx, sin embargo, otros estudios no observaron cambios en el VO2máx a pesar de mejorar el rendimiento del ejercicio aeróbico (Burgomaster et al., 2005-2006) pues los autores encontraron hallazgos que podrían explicarse por un VO2pico relativamente alto al inicio de realizar los estudio (48,7 ml $¥ \mathrm{~kg}-1 ¥ / \mathrm{min})$, lo cual no se obtuvieron en nuestro estudio. Por otra parte al utilizar sujetos con valores de VO2mäx basales de forma similar, se encontró aumentos significativos en el VO2máx después de 2 semanas de entrenamiento anaeróbico (Hazell et al., 2010), el VO2máx aumentó de $4.2 \%$ a $13.4 \%$ en los estudios realizados, las mejoras en el VO2máx pueden explicarse por: (1) mayor disponibilidad de oxígeno debido a efectos centrales (gasto 
cardiaco) y (2) como consecuencia de adaptaciones periféricas con capacidad mejorada para extraer y utilizar oxígeno disponible debido al aumento del potencial oxidativo muscular. Con lo cual se puede afirmar que el tiempo de intervención de nuestros trabajo fue adecuado (seis meses).

En otra investigación se determinó el VO2 máximo y la capacidad de recuperación que tienen los jugadores profesionales y no profesionales de fútbol-sala, la muestra realizó una prueba máxima de esfuerzo para VO2 máx., elegida para determinar el perfil aeróbico y la capacidad de recuperación conocida como "Course Navette" o Test Progresivo de 20 metros ida y vuelta con periodos de 1 minuto de duración; a su vez concordamos con el estudio de (Mocha Bonilla, 2018) quien focalizó el efecto de la aplicación de un programa de intervención para analizar el consumo de oxígeno máximo con la finalidad de observar la condición física. La metodología responde a un diseño cuasi-experimental, es decir, la aplicación de una intervención mediante la toma de datos antes y después, quien también utilizó el test de Course Navette, el cual mide el consumo de oxígeno máximo en cada uno de los participantes. Los resultados muestran avances significativos post intervención, es decir, obtuvieron mejores puntuaciones sobre el VO2máx.; partiendo de esto y según los datos reportados en las figuras 3 y 4 podemos afirmar que nuestro programa obtuvo mejoras significativas en la condición física de la muestra propuesta.

En cuanto a la capacidad de recuperación se puede mencionar que es fundamental en los deportes de prestación mixta: Una buena capacidad de recuperación permitirá realizar una gran cantidad de trabajo a pesar de tener un reducido VO2máx (Álvarez-Medina et al., 2001), datos similares a los encontrados en la tomo inicial de nuestro estudio.

Ramírez-Lechuga et al. (2012) determinaron el efecto de un programa de entrenamiento aeróbico de alta intensidad de 8 semanas, el programa de entrenamiento consistió en la práctica de actividad física aeróbica con una intensidad equivalente al 75-80\% del VO2máx, realizado en 2 y 3 sesiones semanales. Aportando que las tres sesiones empleadas durante la aplicación del programa de entrenamiento aeróbico dan como resultado una mejora en la capacidad aeróbica y la condición física. 
Los estudios (Macpherson et al., 2011; Trilk et al., 2011; Astorino et al., 2012) sobre los efectos centrales y la capacidad oxidativa muscular, los estudios han informado cambios periféricos con adaptaciones enzimáticas mejoradas y aumento de la masa mitocondrial después del entrenamiento, lo que sugiere adaptaciones periféricas significativas que podrían explicar algunas de las mejoras observadas en el VO2máx y el rendimiento aeróbico, lo cual abre las puertas para futuras investigaciones en este campo, tomando como base la aplicación realizada.

\section{Conclusiones.}

$\mathrm{Al}$ inicio de la aplicación de test se notó que la condición física de los árbitros hombres y mujeres se encontraban bajo el promedio para poder rendir y aprobar las pruebas físicas establecidas; pruebas obligatorias que los árbitros deben superar para estar aptos y poder dirigir los encuentros deportivos, por lo cual se propuso un plan de entrenamiento aeróbico.

Se determinó el nivel del consumo máximo de oxigeno (Vo2máx) en los Árbitros Profesionales de Fútbol de Tungurahua, inicialmente se pudo detectar casos de una deficiente condición física y que en algunos casos solo llegaban a muy buena, luego del periodo de entrenamiento aeróbico y tras realizar la nueva valoración del consumo máximo se evidenció una mejora en los datos del (VO2máx), lo cual mejoró la condición física en el grupo de estudio.

Se verificó que el programa de entrenamiento aeróbico es beneficioso en los dos grupos de estudio, sin embargo, las mujeres mejoraron de forma notable, debido a que son una muestra pequeña, mientras que los hombres tuvieron una mejora significativamente por que los grupos se encuentran en una edad de rendimiento físico, lo cual ayudó a optimizar el consumo del volumen máximo de oxígeno, la condición física y el desempeño arbitral.

\section{Referencias bibliográficas.}

Aldana, S., Vereda, F., Hidalgo-Alvarez, R., \& de Vicente, J. (2016). Facile synthesis of magnetic agarose microfibers by directed selfassembly. Polymer, 93, 61-64.

Acosta, F. (2012). Fisiología del Ejercicio. Obtenido de sld.cu: http://www.sld.cu/galerias/pdf/sitios/rehabilitacion-ejer/fisiologiadelejercicio.pdf 
Aerobics org. (2017). aerobics.org. Obtenido de http://www.aerobic.org/

Álvarez-Medina J, Giménez-Salillas L, Manonelles-Marqueta P, Corona Virón P. (2001). Importancia del vo2máx. y de la capacidad de recuperación en los deportes de prestación mixta. Caso práctico: fútbol-sala. Archivos de Medicina del Deporte, 18 (86): $577-83$

Astorino TA, Allen RP, Roberson DW, Jurancich M. Effect of high-intensity interval training on cardiovascular function, VO2max, and muscular force. J Strength Cond Res 2012: 26: 138-145. (s.f.).

Braun, L. (2013). Exercise physiology and cardiovascular fitness. Nurs Clin North Am, 26(1):135-47.

Bumgardner, W. (9 de abril de 2017). Very Well. Obtenido de https://www.verywell.com/what-is-aerobic-metabolism-3432628

Burgomaster KA, Cermak NM, Phillips SM, Benton CR, Bonen A, Gibala MJ. Divergent response of metabolite transport proteins in human skeletal muscle after sprint interval training and detraining. Am J Physiol Regul Integr Comp Physiol 2007: 292: R1970-R197. (s.f.).

Burgomaster KA, Heigenhauser GJ, Gibala MJ. Effect of short-term sprint interval training on human skeletal muscle carbohydrate metabolism during exercise and time-trial performance. J Appl Physiol 2006: 100: 2041-2047. (s.f.).

Casselbury, K. (2017). The History of Aerobics. Chicago: Live Strong. Obtenido de https://www.livestrong.com/article/324355-the-history-of-aerobics/

Cooper, K. H. (2013). En T. Nelson, Can Stress Heal? (pág. 260). Obtenido de https://books.google.com.ec/books?id=k75y6g5-aQAC\&pg=PT40\&redir_esc=y

Hazell TJ, Macpherson RE, Gravelle BM, Lemon PW. 10 Or 30-S sprint interval training bouts enhance both aerobic and anaerobic performance. Eur J Appl Physiol 2010: 110: 153-160. (s.f.).

Losnegard T, Myklebust H, Spencer M, Hallén J. (2012) Variaciones estacionales en VO2max, costo de $\mathrm{O} 2$, déficit de $\mathrm{O} 2$ y rendimiento en esquiadores de élite de fondo. J Strength Cond Res. 2012 Sep 19. (s.f.). 
Macpherson RE, Hazell TJ, Olver TD, Paterson DH, Lemon PW. Run sprint interval training improves aerobic performance but not maximal cardiac output. Med Sci Sports Exerc 2011: 43: 115-122. (s.f.).

Mocha Bonilla, J. A. (2018). Análisis del consumo máximo de oxígeno (vo2máx) post intervención de un programa en juegos pre-deportivos. Ciencia Digital, 2(2), 229244. doi:https://doi.org/10.33262/cienciadigital.v2i2.85

Muñoz R, Calderón M, Paul Medina-González P, Martínez-Saavedra N, Escobar-Cabello M. (2018). Confiabilidad en la medición de la presión inspiratoria máxima y de la capacidad inspiratoria de un fisioterapeuta en entrenamiento. Fisioter Pesqui; 25(4):444-451.

Norman, G. (9 de Abril de 2017). Aerobic Metabolism and Exercise. Obtenido de https://www.verywell.com/what-is-aerobic-metabolism-3432628.

Ramírez-Lechuga J, Muros-Molina JJ, Morente-Sánchez J, Sánchez-Muñoz C, y ZabalaDíaz M. (2012). Efecto de un programa de entrenamiento aeróbico de 8 semanas durante las clases de educación física en adolescentes. Nutr Hosp. 2012; 27:747-754

Rivera, H. (8 de abril de 2016). Thougth CO. Obtenido de https://www.thoughtco.com/aerobic-exercise-definition-415261

Rivera, J. (2011). Alto Rendimiento: La adaptación y la excelencia deportiva. j. Sports, 327329.

Sport-fitness. (2014). Fisiología del Ejercicio. Obtenido de VO2 máx., Potencia aeróbica y consumo máximo de oxígeno: https://www.sport-fitness-advisor.com/vo2max.html

Trilk JL, Singhal A, Bigelman KA, Cureton KJ. Effect of sprint interval training on circulatory function during exercise in sedentary, overweight/obese women. Eur J Appl Physiol 2011: 111: 1591-1597. (s.f.).

\section{【Ciencia}




\section{PARA CITAR EL ARTÍCULO INDEXADO.}

Jiménez Ruiz, L., Caguana Caguana, J., Garcés Duran, S., \& Calderón Sánchez, A. (2019). Entrenamiento Aeróbico y el Consumo Máximo de Oxígeno (Vo2máx) en árbitros

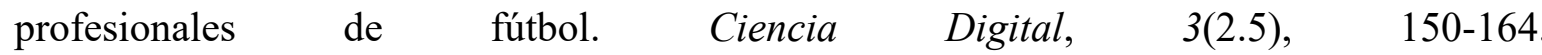
https://doi.org/10.33262/cienciadigital.v3i2.5.539

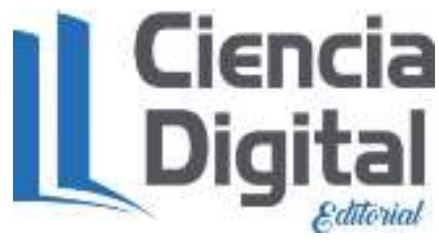

El artículo que se publica es de exclusiva responsabilidad de los autores y no necesariamente reflejan el pensamiento de la Revista Ciencia Digital.

El artículo queda en propiedad de la revista y, por tanto, su publicación parcial y/o total en otro medio tiene que ser autorizado por el director de la Revista Ciencia Digital.
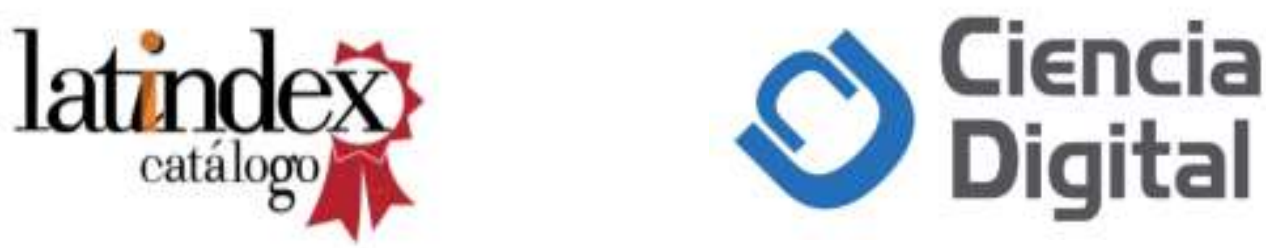